\title{
Letramento multimodal: participação em gêneros discursivos multimodais em livros didáticos de língua inglesa
}

Nathalia Rodrigues Catto

\author{
Universidade Federal de Santa Maria
}

\begin{abstract}
Resumo
O presente trabalho explora de que maneira duas coleções aprovadas pelo Programa Nacional do Livro Didático promovem letramento multimodal no ensino de língua inglesa. Foram analisadas atividades de produção e compreensão escrita com ênfase especial em algum dos gêneros dos quadrinhos, como tira em quadrinhos ou história em quadrinhos.
\end{abstract}

Palavras-Chave: Letramento multimodal, Livro didático de língua inglesa, Análise Crítica de Gênero.

\begin{abstract}
This paper explores how two textbook collections approved by the "Programa Nacional do Livro Didático" promote multimodal literacy in English language teaching. Written production and comprehension activities were analyzed with special emphasis on the genres of comics, such as comic strips.
\end{abstract}

Keywords: Multimodal literacy, English textbook, Critical Genre Analysis.

\section{INTRODUÇÃO}

A área de Linguística Aplicada no Brasil cresce expansivamente em busca de respostas para questões pertinentes à compreensão sobre como a linguagem funciona, em contextos variados. Um dos contextos amplamente investigados é o pedagógico, representado em grande parte pelos materiais didáticos. Inúmeros estudos exploram o papel do livro didático como recurso para o ensino de linguagem. No entanto, especialmente sobre o livro didático de língua estrangeira, um limitado número de pesquisas compõe o campo em questão, visto que a popularização desses materiais na rede pública de ensino é extremamente recente. Não obstante, um olhar multimodal sobre tais materiais é ainda pouco explorado, o qual caracteriza a presente pesquisa.

Estudos recentes sobre letramento (New London Group, 1996; Cope \& Kalantzis, 2000, 2008; Kress, 2003; Dionísio, 2005) têm apontado que a participação bem sucedida nas práticas sociais contemporâneas, marcadas pela diversidade cultural, linguística e 
tecnológica, exige múltiplos saberes, cada vez mais especializados. No contexto escolar, essa demanda convoca pedagogias que deem conta dessa diversidade. Tal é o caso da pedagogia dos multiletramentos, proposta pelo New London Group (1996; Cope \& Kalantzis, 2000) como alternativa de ensino que permite inserir os alunos em contextos culturais variados e, para isso, desenvolver habilidades para expandir o acesso a determinados contextos (Cope \& Kalantzis, 2000). Compreender o ensino de linguagem de tal maneira demanda, sobretudo, contemplar uma ampliação no foco sobre a linguagem como gênero, abrangendo a materialização multimodal dos eventos comunicativos que medeiam as práticas sociais contemporâneas.

O modelo dos multiletramentos destaca dois argumentos importantes e correlacionados na reavaliação sobre os letramentos (no plural), múltiplos em dois sentidos: 1) na diversidade cultural e linguística; e 2) na influência de novas tecnologias comunicativas (Cope \& Kalantzis, 2008, p. 197). O primeiro inclui as múltiplas variedades linguísticas e culturais nos âmbitos profissional, nacional, escolar, e o segundo contempla a multimodalidade como inerente às formas contemporâneas de representação e aos novos espaços por meio dos quais interagimos. É no segundo argumento que o presente trabalho se concentra ${ }^{1}$, ou seja, sobre uma questão fundamental a ser considerada nesse sentido: em que medida e como os saberes que fazem parte do letramento multimodal são recontextualizados em livros didáticos de língua inglesa. Em vista da lacuna na literatura sobre como compreender a relação entre teoria e prática, ou seja, sobre como os conhecimentos do âmbito científico são recontextualizados para o âmbito pedagógico, pretendo, no presente trabalho, analisar em que medida e como o conceito de letramento multimodal é recontextualizado em livros didáticos de língua inglesa.

Uma vez que pesquisas prévias desenvolvidas por esta pesquisadora (Catto \& Hendges, 2010; Catto, 2012) exploravam o funcionamento de tiras em quadrinhos, busco no presente trabalho analisar em duas coleções de livros didáticos aprovados pelo Programa Nacional do Livro Didático atividades de produção e compreensão escrita cujo objeto de análise seja composto por algum dos gêneros dos quadrinhos, qual seja charge, cartum, tira

\footnotetext{
${ }^{1}$ Reconheço a importância do primeiro argumento, no entanto, o espaço e tempo limitados e os objetivos do presente trabalho impedem uma discussão detalhada sobre tal aspecto. Sugiro uma análise aprofundada de ambos os pontos em trabalhos futuros.
} 
em quadrinhos ou história em quadrinhos para explorar de que maneira essas coleções promovem letramento multimodal no ensino de língua inglesa.

Além disso, o presente trabalho visa verificar em que medida essas coleções recontextualizam tal conceito e revelam o potencial pedagógico das diversas linguagens no ensino de língua inglesa. Tendo em vista a relevância dessas questões para o ensino de inglês, procuro discutir como esses recursos semióticos podem ser explorados na formação de sujeitos multiletrados.

\section{FUNDAMENTAÇÃO TEÓRICA}

A presente pesquisa está situada na grande área de Linguística Aplicada, especificamente embasada nos pressupostos teórico-metodológicos da Análise Crítica de Gênero (Meurer, 2002, 2004; Bhatia, 2004; Motta-Roth, 2005, 2008). A ACG cruza, mas também expande conceitual e metodologicamente, escolas de gênero como a Sóciorretórica (Miller, 1984; Bazerman, 1988; Swales, 1990, 2004; Bhatia, 1993, 2004), uma perspectiva sócio-semiótica sobre a linguagem a partir da Linguística sistêmico-funcional (Halliday \& Hasan, 1985/1989; Martin, 1985/1989; Halliday, 1994; Halliday \& Matthiessen, 2004), combinadas com o componente crítico de análise, por meio da Análise Crítica do Discurso (Fairclough, 1992, 2003; van Leeuwen, 1996, 2008). Nesse sentido, a configuração metodológica da presente pesquisa está orientada sob esse paradigma, pelo fato de entrelaçar diferentes bases teóricas para dar conta de uma análise abrangente sobre a configuração textual e contextual do livro didático como mediador das práticas sociais do contexto pedagógico.

\section{METODOLOGIA}

O universo de análise da presente pesquisa corresponde ao volume 1 de duas coleções de livros didáticos de língua inglesa, recomendadas pelo PNLD e o papel da multimodalidade para o ensino de língua inglesa nesses recursos didáticos. As duas coleções selecionadas, aprovadas pelo PNLD de 2012 para o Ensino Médio são: Prime Inglês para o Ensino Médio (Dias, Jucá \& Faria, 2010), e English for all (Aun, Moraes \& Sansanovicz, 2010), representadas no Guia do Livro Didático (Brasil, 2011) pelos códigos 25149COL33 e 25056COL33, respectivamente. Esses dois títulos foram escolhidos dentre os sete aprovados pelo PNLD por atenderem em maior escala aos critérios de avaliação 
especificados no edital de convocação para seleção de obras didáticas para o PNLD 2012 do Ensino Médio e descritos no Guia do Livro Didático (Brasil, 2011). Dessa maneira, as duas coleções selecionadas apresentam ao mesmo tempo maior número de critérios bem avaliados e menor número de deficiências nos critérios organizadores estabelecidos pelo PNLD.

Considerando os exemplares do livro do aluno, foram analisados os enunciados das atividades didáticas (identificados por números cardinais na coleção Prime e por letras maiúsculas na coleção English for all) bem como algumas questões dessas atividades (identificadas por letras minúsculas no Prime e por números no English for all). A investigação foi além do enunciado da atividade à medida que as questões contempladas nas atividades se mostraram relevantes para os objetivos da análise. Dessa maneira, as questões que faziam referência à linguagem não verbal imagética foram tomadas como evidência para explicar como as atividades exploram o papel dessa linguagem em gêneros multimodais para o ensino de língua inglesa. Os dados foram interpretados à luz da literatura prévia sobre multimodalidade, ensino de língua inglesa e sobre os aspectos que caracterizam o conceito contemporâneo de letramento multimodal. Tal embasamento teórico será adotado no presente trabalho para compreender e explicar como conhecimentos do letramento multimodal são explorados nas atividades didáticas, considerando a relação entre tipo de atividade e aspecto do letramento multimodal explorado.

\section{Conhecer, selecionar e combinar de maneira informada e qualificada os múltiplos modos semióticos}

a) no nível léxico-gramatical, mobilizando as potencialidades do sistema linguístico verbal e visual;

b) no nível semântico e pragmático, mobilizando o processo de produção de sentido;

c) no nível do registro, mobilizando particularidades do contexto de situação;

d) no nível do gênero, mobilizando configurações textuais e contextuais recorrentes;

e) no nível ideológico, mobilizando análise crítica dos diversos Discursos que atravessam os textos.

Quadro 1: Conhecimentos centrais na literatura contemporânea contemplados no letramento multimodal e no letramento visual.

Cada um dos estratos da linguagem, conforme proposta de Motta-Roth (2008) para a estratificação dos níveis da linguagem, baseada em Hendges (2005) e Martin (1992), incorpora aspectos os quais devem estar previstos nas atividades didáticas cuja 
representação de linguagem corresponde ao conceito de gênero discursivo. No nível da ideologia, as atividades didáticas deveriam explorar aspectos relacionados à inclusão/exclusão de participantes, representações e vozes atribuídas no texto. No nível do gênero ou do contexto de cultura, aspectos relacionados aos elementos recorrentes das práticas sociais bem como características textuais e contextuais deveriam ser consideradas. No nível do contexto de situação, estariam previstos aspectos relacionados ao assunto, o campo, quem são os participantes, as relações, e o modo como a situação acontece. No nível da semântica e pragmática, as atividades deveriam contemplar a análise dos significados relevantes atualizados em determinado gênero, considerando o conteúdo da experiência. Já no nível léxico-gramatical, o foco das atividades estaria nas escolhas em termos de vocabulário e gramática, ou seja, no sistema linguístico atualizado no texto.

Dessa forma, as atividades didáticas foram selecionadas considerando aquelas que demandassem para a resolução das tarefas propostas: a) a análise de todas as linguagens (verbal e não verbal imagética) e b) a análise da linguagem não verbal imagética apenas. Em seguida, as atividades foram analisadas a fim de identificar o estrato da linguagem explorado: a) nível léxico-gramatical; b) nível semântico e pragmático; c) nível do contexto de situação ou registro; d) nível do contexto de cultura ou gênero; e e) nível do Discurso, da ideologia. Por fim, foram estabelecidas relações entre cada um dos conhecimentos do letramento multimodal e os índices linguísticos que remetem a esses conhecimentos nas atividades didáticas do corpus.

Estudos prévios (Arnt, 2012; Kummer, 2012; Rossi, 2012) e análises piloto em busca da identificação do papel da linguagem não verbal imagética aliada à linguagem verbal em materiais didáticos revelaram traços ricos em significação para essa investigação, marcados, por exemplo, por processos nos enunciados das atividades, referência léxicogramatical específica que caracteriza a linguagem não verbal imagética e natureza da exploração, ou seja, que função da linguagem não verbal imagética dos gêneros multimodais é explorado nas atividades didáticas (função ideacional, interpessoal e/ou textual).

As etapas previamente descritas tiveram como ferramentas analíticas a Gramática Sistêmico-Funcional (Halliday, 1994; Halliday \& Matthiessen, 2004) e a Gramática Visual 
(Kress \& van Leeuwen, 1996, 2006) para descrever os enunciados e as questões das atividades didáticas e as imagens relacionadas a estas, respectivamente.

\section{RESULTADOS}

As atividades contemplam em grande parte os diferentes níveis da linguagem. No entanto, há um predomínio de atividades que exploram a linguagem nos gêneros dos quadrinhos no nível do registro, considerando o conteúdo da experiência no contexto de situação, e de atividades que exploram o nível da semântica e pragmática da linguagem, em relação às estruturas semióticas de sentido e uso.

Linguagens analisadas

\section{Linguagem verbal + não verbal imagética}

Linguagem não verbal imagética
EFA 1

6 atividades

4 atividades

10
PR 1

TOTAL

TOTAL DE ATIVIDADES
32 atividades

21 atividades

53
38

25

\begin{tabular}{cccc} 
TOTAL & 10 & 53 & 63 \\
DE ATIVIDADES & & \\
\hline Tabela 1: Linguagens analisadas nas atividades do corpus $^{2}$
\end{tabular}

Tabela 1: Linguagens analisadas nas atividades do corpus ${ }^{2}$

Sobre os conhecimentos do letramento multimodal, tanto a coleção English for all (6) quanto a coleção Prime (32) apresentam 60\% das atividades voltadas para a análise de ambas as linguagens a fim de resolver as tarefas propostas. A maioria das atividades contempla esses conhecimentos no nível da léxico-gramática, do registro e do gênero.

No nível da léxico-gramática, as atividades didáticas exploram elementos relacionados à linguagem típica dos quadrinhos, em termos de balões de fala, por exemplo, e a habilidade de identificação de elementos na imagem, marcada pelo Processo "identify" em destaque no Quadro 2. Na coleção EFA1, este estrato da linguagem é o mais explorado nas atividades.

\footnotetext{
2 A abreviação "PR1" e "EFA1" foi adotada para fazer referência aos volumes 1 das duas coleções, Prime e English for all, respectivamente.
} 


\begin{tabular}{lccc}
\multicolumn{1}{c}{ ESTRATO DA } & EFA 1 & PR 1 & TOTAL \\
\hline LINGUAGEM & 4 atividades & 7 atividades & $\mathbf{1 1}$ \\
Semântica e Pragmática & 2 atividades & 17 atividades & $\mathbf{1 9}$ \\
Registro & 1 atividade & 20 atividades & $\mathbf{2 1}$ \\
Gênero & 1 atividade & 4 atividades & $\mathbf{5}$ \\
Ideologia & 2 atividades & 5 atividades & $\mathbf{7}$ \\
\multicolumn{1}{c}{ TOTAL DE ATIVIDADES } & $\mathbf{1 0}$ & $\mathbf{5 3}$ & $\mathbf{6 3}$ \\
\hline
\end{tabular}

Tabela 2: Estrato da linguagem explorado, relacionado ao número de atividades em cada coleção analisada.

\section{PR1}

5. Look back at the comic strips and answer the questions.

a. What electronic gadgets can you identify in the illustrations?

\section{PR1}

1. Look at the bubbles and match the meanings with the circled resources the letterer used.

\section{PR1}

2. Match the bubbles to their function.

a. thought bubble c. burst bubble

b. whispering bubble $\mathrm{d}$. telepathic bubble

Quadro 2: Exemplos de atividades do corpus relacionadas ao nível da léxico-gramática

No nível da semântica e pragmática, estrato amplamente explorado nas atividades analisadas, foram identificados elementos relacionados à noção de sentido e ao contexto de uso da léxico-gramática na comunicação, como expressões adequadas ao sentido pretendido no texto, compreensão e interpretação de elementos linguísticos e relacionados aos objetivos do gênero analisado. 


\section{EFA1}

B. Underline the correct words according to the text.

This is a story about a little boy / girl named Calvin / Hobbes.

His father / mother is dreaming / waking him up.

\section{EFA1}

E. O que você acha que Calvin quis dizer com "My dreams are getting way too literal"?

\section{PR1}

2. Look at this cartoon. Hold a debate around the questions below.

a. Do you think it's funny? Why (not)?

c. In your opinion, what seems to be the girl's strongets intelligence?

Quadro 3: Exemplos de atividades do corpus relacionadas ao nível da semântica e pragmática

No nível do registro, foram identificadas atividades que exploram aspectos relacionados ao campo - o assunto, às relações - quem são os participantes, e ao modo como a situação se realiza, marcados por elementos como "who", "about", "purpose" e “where” e pela análise do papel das linguagens verbal e não verbal imagética na realização do objetivo do gênero.

\section{PR1}

1. Look at the comic strip below and answer the questions.

b. Who are they?

c. What is the comic strip about?

d. What is its purpose?

\section{PR1}

3. Answer the following questions about the cartoon above.

a. Where does the scene take place?

b. How many characters are there? Who are they? What are they doing?

\section{PR 1}

b. How important is the combination of verbal and non-verbal language to express this cartoon's funny element? Explain your answer.

Quadro 4: Exemplos de atividades do corpus relacionadas ao nível do registro 
No nível do gênero, as atividades didáticas do corpus exploram características da prática social, em termos de recorrência. Na coleção Prime, há referência explícita a esse nível por meio da subseção "Genre Analysis”. Índices linguísticos como "characteristics" e "characterize" e explicitamente "genre" apontam para a análise da linguagem sob esse prisma.

\section{PR1}

1. In some of the boxes below, you can find characteristics of a newspaper editorial cartoon. Check the ones you think are correct.

\section{PR1}

2. Look at the comic strip on page 144 and consider your experience with this genre. Use the cues below to characterize it. Write sentences and use frequency adverbs when possible.

\section{EFA1}

B. Que características desse tipo de texto ajudam a compreender melhor a história?

Quadro 5: Exemplos de atividades do corpus relacionadas ao nível do gênero

No nível da ideologia, elementos como "included", "able to use" e "who speaks" direcionam para uma discussão que desnaturaliza situações de senso comum, fazendo o aluno observar questões pertinentes aos diferentes discursos que perpassam os textos.

\section{PR1}

c. What's the main message in editorial cartoon B?

d. Is Brazil included in this message?

\section{PR1}

b. Were you able to use a computer when you were a child?

d. What about your parents - which devices could they use?

\section{PR1}

\section{c. Who speaks? How many times?}

Quadro 6: Exemplos de atividades do corpus relacionadas ao nível do discurso 
As orientações explícitas ao aluno sobre o que fazer ou como responder às atividades considerando a multimodalidade dos gêneros encontram-se essencialmente nos elementos denominados "Tip" e "Be aware”, presentes apenas na coleção Prime.

\section{CONSIDERAÇÕES FINAIS}

Os resultados indicam que grande parte das atividades didáticas exploram conhecimentos do letramento multimodal por meio da produção e, em especial, da análise de gêneros multimodais. Ainda assim acredito que devido às discussões recentes propostas para e sobre o letramento multimodal e os multiletramentos no Brasil ainda são necessárias ações pedagógicas explícitas em busca do desenvolvimento de uma "competência comunicativa multimodal" (Royce, 2007; Heberle, 2010 apud Nascimento, Bezerra \& Heberle, 2011) em livros didáticos. Para tanto, se faz necessário explicitar os procedimentos por meio dos quais as atividades contemplariam os saberes necessários para a participação informada em gêneros discursivos multimodais. Em última instância, o presente trabalho pretende oferecer subsídios para a abordagem e discussão de aspectos multimodais em livros didáticos para o ensino de línguas, levando em consideração "toda a complexidade desse objeto cultural” (Bunzen, 2005, p. 12).

\section{REFERÊNCIAS}

Arnt, J. T. (2012). Análise de atividades didáticas com vistas à promoção de letramento científico. 137 f. Dissertação (Mestrado em Letras) - Universidade Federal de Santa Maria, Santa Maria.

Aun, E., Moraes, M. C. P. \& Sansanovicz, N. B. (2010). English for all. São Paulo: Saraiva.

Bazerman, C. (1988). Shaping written knowledge. Madison: The University of Wisconsin Press.

Bhatia, V. (1993). Analysing genre: language use in professional settings. London: Longman.

Bhatia, V. (2004). Worlds of Written Discourse. London: Continuum.

Brasil. MEC/SEF. (2011). Guia de Livros Didáticos PNLD 2012: Língua Estrangeira Moderna - Ensino Médio. Brasília: Ministério da Educação/Secretaria de Educação Básica. 
Bunzen, C. (2005). Livro didático de Língua Portuguesa: um gênero do discurso. $168 \mathrm{f}$. Dissertação (Mestrado em Linguística Aplicada) - Departamento de Lingüística Aplicada, Instituto de Estudos da Linguagem, Universidade Estadual de Campinas, Campinas.

Catto, N. R. (2012). Uma análise crítica do gênero multimodal tira em quadrinho: questões teóricas, metodológicas e pedagógicas. 123f. Dissertação (Mestrado em Letras) Universidade Federal de Santa Maria. Santa Maria.

Catto, N. R. \& Hendges, G. R. (2010). Análise de gêneros multimodais com foco em tiras em quadrinho. Signum: Estudos de Linguagem, 13 (2), 193-217.

Cope, B. \& Kalantzis, M. (2008). Language education and multiliteracies. In: May, S. \& Hornberger, N. H. (eds.). Encyclopedia of Language and Education, (1), 195-211.

Cope, B. \& Kalantzis, M. (2000). Multiliteracies: literacy learning and the design of social futures. London: Routledge.

Creswell, J. W. (1994). Research design: qualitative and quantitative approaches. London: Sage Publications.

Dias, R., Jucá, L. \& Faria, R. (2010). Prime - Inglês para o Ensino Médio. São Paulo: Macmillan.

Dalacorte, M. C. F. (1994). An attempt to analyse comprehension questions of a reading passage. Signótica, 6, 91-102.

Dionísio, A. P. (2006). Gêneros multimodais e multiletramento. In: Karwoski, A. M., Gaydeczka, B. \& Brito, K. S. (eds.) Gêneros textuais: reflexões e ensino. 131-144. Rio de Janeiro: Lucerna.

Fairclough, N. (2003). Analysing discourse: textual analysis for social research. London/New York: Routledge.

Fairclough, N. (1992). Discourse and social change. Cambridge: Polity Press.

Halliday, M. A. K. (1994). An introduction to functional grammar. London: Arnold.

Halliday, M. A. K. \& Hasan, R. (1989). Language, context, and text: Aspects of language in a social-semiotic perspective. Oxford: Oxford University Press.

Halliday, M. A. K. \& Matthiessen, C. M. I. M. (2004). An introduction to functional grammar. 3 ed. London: Arnold.

Hendges, G. R. (2005). A genre and register analysis of electronic research articles from a systemic functional perspective: new medium, new meanings. Projeto de doutorado. Florianópolis: PPGI/UFSC.

Kress, G. (2003). Literacy in the new media age. London/New York: Routledge. 
Kress, G. \& van Leeuwen, T. (1996). Reading images: the grammar of visual design. London: Routledge.

Kress, G. \& van Leeuwen, T. (2006). Reading images: the grammar of visual design. London: Routledge.

Kummer, D. (2012). O livro didático de língua inglesa: uma abordagem multimodal. 53f. Monografia (Especialização em Linguagem e Representação) - Centro Universitário Franciscano. Santa Maria.

Martin, J. E. (1985/1989). Factual writing: Exploring and challenging social reality. Oxford: Oxford University Press.

Meurer, J. L. (2004). Ampliando a noção de contexto na Linguística Sistêmico-Funcional e na Análise Crítica do Discurso. Linguagem em (Dis)curso, 4, 133-157.

Meurer, J. L. (2002). Genre as diversity, and rhetorical mode as unity in language use. Ilha do Desterro, 43 (0), 61-82.

Miller, C. (1984). Genre as social action. Quarterly Journal of Speech, 70, 151-167.

Moita Lopes, L. P. (1994). Pesquisa interpretativista em Lingüística Aplicada: a linguagem como condição e solução. D.E.L.T.A, 10 (2), 329-338.

Motta-Roth, D. (2003). A dinâmica de produção de conhecimento: teoria e dados, pesquisador e pesquisados. Revista Brasileira de Linguística Aplicada, 3 (1), 165-177.

Motta-Roth, D. (2008). Análise crítica de gêneros: contribuições para o ensino e a pesquisa de linguagem. D.E.L.T.A, 24 (2), 341-383.

Motta-Roth, D. (2006). Questões de metodologia em análise de gênero. In: Karwoski, A. M., Gaydecka, B. \& Brito, K. S. (eds.). Gêneros textuais: reflexões e ensino. 179-202. Rio de Janeiro: Lucerna.

Nascimento, R. G., Bezerra, F. A. S. \& Heberle, V. M. (2011). Multiletramentos: iniciação à análise de imagens. Linguagem \& Ensino, 14 (2), 529-552.

New London Group. (1996). A pedagogy of multiliteracies: designing social futures. Harvard Educational Review, 66 (1), 60-92.

Oliveira, S. (2000). Question-asking in Brazilian Portuguese reading comprehension textbooks. Ilha do Desterro, 38 (1), p. 039-056.

Rossi, A. F. E. (2012). Recontextualização do discurso da ciência da linguagem em livros didáticos de língua inglesa. 139f. Dissertação (Mestrado em Letras) - Universidade Federal de Santa Maria, Santa Maria. 
Royce, T. (2007). Inter-semiotic complementarity: A framework for multimodal discourse analysis. In: Royce, T., Bowcher, W. L. New directions in the analysis of multimodal discourse. 63-109. New Jersey: Lawrence Erlbaum Associates.

Swales, J. (1990). Genre analysis: English in academic and research settings. Cambridge: Cambridge University Press.

Swales, J. (2004). Research genres: exploration and applications. Cambridge: Cambridge University Press.

van Leeuwen, T. (2008). Discourse and practice: new tools for critical discourse analysis. New York: Oxford.

van Leeuwen, T. (1996). The representation of social actors. In: Caldas-Coulthard, C. R. \& Coulthard, M. (eds.). Texts and practice. 32-70. London: Routledge.

\section{A AUTORA}

Nathalia Rodrigues Catto é licenciada em Letras Inglês pela Universidade Federal de Santa Maria (UFSM) e Mestre em Letras pelo Programa de Pós-graduação em Letras, com ênfase em Estudos Linguísticos, pela mesma instituição. Atualmente é Professora do Ensino Básico, Técnico e Tecnológico no Instituto Federal Farroupilha Campus Alegrete, na área de língua inglesa, e doutoranda do Programa de Pós-graduação em Letras da UFSM. Seus interesses de pesquisa contemplam multimodalidade/multiletramentos, letramento multimodal, análise crítica de gêneros, livros didáticos, ensino de línguas e tiras em quadrinhos.

E-mail: nathcatto@gmail.com 